\title{
Design of urban public transport lines as a multiple criteria optimisation problem
}

\author{
L. Jánošíková ${ }^{1}$, M. Blatoň ${ }^{2} \&$ D. Teichmann ${ }^{2}$ \\ ${ }^{I}$ Department of Transportation Networks, \\ Faculty of Management Science and Informatics, University of Žilina, \\ Slovak Republic \\ ${ }^{2}$ Institute of Transport, Faculty of Mechanical Engineering, \\ VŠB-Technical University of Ostrava, Czech Republic
}

\begin{abstract}
The paper describes a mathematical programming approach used for the lineplanning problem in urban public transport. The input data include the transportation network in a city, O-D matrix of travel demand, and the set of available vehicles of specified transportation modes and types. The goal of line planning is to design the routes of lines and their frequencies. Supposing an initial set of lines has been proposed, the line-planning problem is formulated and solved as a multiple criteria optimisation problem, where the criteria reflect the travellers' demand for a high quality service, the operator's interest in an effective service, and the environmental impact of the vehicles. The solution to this problem specifies the number of vehicles of the given mode and type operating on the lines. Lines, which are not assigned a vehicle, will not operate. At the same time, the solution specifies optimal passenger routes in the line network. Then an iterative process follows which computes new line frequencies using a discrete choice model to respect passengers' behaviour when they have multiple travel alternatives.
\end{abstract}

Keywords: line-planning problem, mathematical programming, multiple criteria optimisation, discrete choice model.

\section{Introduction}

Design of urban public transport lines should be addressed in the context of public transport planning process, which consists of five stages [3]: 
1. design of line routes,

2. frequency setting,

3. timetable development,

4. vehicle scheduling,

5. driver scheduling.

The first two steps have decisive influence on the quality of the whole transportation system. Line routes and frequencies determine the level to which the transportation demand is satisfied because they have direct impact on the spatial and time accessibility of the transportation service. The frequency of a line indicates the basic timetable period and controls the transportation capacity of the line. The route length determines operating costs on the line and together with the frequency indicates the number of vehicles needed to operate on the line, which further results in investment costs. Thus line routes and frequencies are independent variables which influence two main objectives of the public transport design, namely the quality of the service perceived by users and the operator costs.

In several previous approaches (e.g. [2]) the first two stages of the design (line routing and frequency setting) were solved simultaneously and referred to as the line-planning problem (LPP). In our research we have followed this line of research considering the LPP as a multiple criteria optimization problem. We have proposed a new solution procedure which combines a mathematical programming approach with a trip assignment procedure based on a discrete choice model. This paper presents a mathematical programming formulation of the multi-criteria LPP and the solution procedure.

\section{Problem statement}

The problem can be stated as follows. Assume that the travel demand is given in the form of a so-called origin-destination matrix (OD matrix). An element of the OD matrix determines the value of the transportation flow from the origin to the destination expressed in the number of travellers per time unit, e.g. an hour. Furthermore, an initial set of all feasible lines has been pre-defined. Because the line design is not usually performed in an "empty" city without any public transport, but rather is regarded as re-engineering of an existing transportation system, this set of feasible lines can comprise current lines, modified current lines (e.g. lines avoiding overloaded roads), as well as new lines corresponding to the shortest routes between origin and destination nodes. The task is to determine which lines from the candidate set will operate and to set their frequencies so that the quality of the transportation service can be maximal and the total operator costs minimal.

The optimisation criteria should be stated more precisely. First let us look at the quality of the transportation service from the passengers' point of view. Two main aspects of the transportation service perceived by users are time and cost. User cost (fare) does not play a role in urban line planning because:

- Demand is treated as fixed and independent on the service quality offered between any origin-destination pair. It means that modal split is supposed 
to be done before and we deal only with those people who have decided for public transport, so only the portion of the travel demand, which is related to public transport, inputs the problem.

- Costs of all urban lines are supposed to be the same.

So we can omit user cost and focus on time as the only passenger criterion. If travel demand refers to public transport stops, then travel time consists of three components: in-vehicle time, origin waiting time (time that a passenger waits for a bus at the origin stop), and transfer waiting time (time that a passenger waits during transfers). In-vehicle time depends proportionally on line routes. Waiting times depend on line frequencies, but this latter dependency is inverse proportional, which would result in a non-linear mathematical model [6]. This is one of the reasons why waiting times are not included in our mathematical programming model. Another reason will be stated in the next section.

The second criterion of the LPP is operator costs, which can be divided into operating and investment costs. As was explained in the introduction, these costs depend directly on route lengths and line frequencies.

Another goal of the line design not mentioned so far can be to minimize negative environmental impact of the public transport, particularly energy consumption and air pollution [1].

\section{Mathematical model}

Input to the model includes a transportation network, travel demand, available vehicle fleet, and candidate lines.

A transportation network is modelled by a (di)graph $G=(N, A, \boldsymbol{t})$, where $N$ is a set of nodes (stops and road junctions) and $A$ is a set of feasible links, i.e. streets that candidate lines pass through. Every link $a \in A$ is associated with a time distance $t_{a}$; a vector of time distances is denoted by symbol $\boldsymbol{t}$.

The travel demand is represented by an OD matrix $\boldsymbol{P}=\left\{p^{r s}\right\}$, where $p^{r s}$ indicates the number of travellers who want to travel in the considered time horizon (e.g. an hour) from the origin stop $r$ to the destination stop $s$. The set of all OD pairs $(r, s)$ is denoted by symbol $Q$.

Vehicles of various types and sizes can operate in the network. Let $I$ denote the set of available vehicle types (e.g. bus, trolley bus, tram). Vehicles of a given type can have different capacities. Let $J_{i}$ stand for the set of possible sizes for vehicle type $i \in I$. A vehicle of type $i$ with size $j$ has capacity $k_{i j}$ seat places and an operator can use at most $n_{i j}$ of these vehicles. Further, vehicles differ in energy consumption, engine emissions, operating and investment costs. To make the model easy to understand and its explanation simpler, we confine to bus traffic and consider only one air pollutant, e.g. carbon monoxide CO. The bus type is specified by the drive, which may be diesel or Compressed Natural Gas $(\mathrm{CNG})$. Let $e_{l i j}$ be the amount of $\mathrm{CO}$ (given in gram) produced by one bus of drive type $i$ and size $j$ during one turn-round on line $l$. The acquisition of one bus of drive type $i$ and size $j$ is connected with investment costs $d_{i j}$ [€.bus $\left.{ }^{-1}\right]$. One hour operation of a bus of type $i$ and size $j \operatorname{costs} c_{i j}\left[€ \cdot\right.$ bus $\left.^{-1} \cdot \mathrm{h}^{-1}\right]$. 
Let $L$ stand for the initial set of all feasible lines. Line $l \in L$ is characterised by its route and a turnaround time $t_{l}^{\text {turn }}$ which is the time it takes to drive line $l$ including stopping times at stops and lay-over time at the terminal point. Turnaround time is assumed to be independent on the vehicle size or loading.

The goal is to decide which lines from the candidate set will operate and to set their frequencies. This decision is modelled by integer variables $x_{l i j}$ which determine how many vehicles of type $i$ and size $j$ will operate on line $l$. Line $l$ that is not assigned a vehicle in the optimal solution (i.e. $x_{l i j}=0$ for all $i \in I$, $j \in J_{i}$ ) will not operate. In terms of these variables, frequency $f_{l}$ of line $l$ can be calculated as follows:

$$
f_{l}=\frac{1}{t_{l}^{\text {turn }}} \sum_{i \in I} \sum_{j \in J_{i}} x_{l i j}
$$

A common approach reported in the literature (e.g. in $[4,11,12])$ fixes travel paths of the passengers before the lines are proposed. For every pair $(r, s)$, a shortest path in the transportation network is computed. The result of this preprocessing step is the total number of passengers passing through every link in the given time period (the value of the flow on the link), which inputs the model as a parameter. The model contains a set of constraints assuring that the proposed lines cover with a sufficient capacity all those links, which are used by at least one passenger. Our model utilizes a different approach (proposed in [2]), where optimal passengers' routes are set during the solution process, i.e. assignment of passenger flows on the links is no more an input to the model but it becomes an output (a decision). This decision is represented by variables $y_{a}^{r s} \in R_{0}^{+}$, which define how many passengers from $r$ to $s$ pass through link $a$. The total in-vehicle time that passengers spend travelling from $r$ to $s$ is expressed by the following term:

$$
\sum_{a \in A} t_{a} y_{a}^{r s}
$$

However, waiting times cannot be modelled because we do not know in advance the number of travellers who board or change particular lines at particular stops.

A common assumption applied in real traffic is that every line can be served only by one vehicle type [13]. To model this assumption, we introduce auxiliary binary variables $z_{l i}$. Value 1 of variable $z_{l i}$ indicates that vehicles of type $i$ operate on line $l$, otherwise $z_{l i}$ is 0 .

Three other symbols occur in the following model:

$L_{a}$ - a set of lines passing through link $a$;

$A_{v}^{+}$- a set of links outgoing node $v$;

$A_{v}^{-}-$a set of links ingoing node $v$. 
Now the mixed integer programming model for the line planning problem with multiple criteria can be written as:

$$
\begin{gathered}
\text { minimise } \sum_{i \in I} \sum_{j \in J_{i}} d_{i j} \sum_{l \in L} x_{l i j} \\
\text { minimise } \sum_{i \in I} \sum_{j \in J_{i}} c_{i j} \sum_{l \in L} t_{l}^{t u r n} x_{l i j} \\
\text { minimise } \sum_{i \in I} \sum_{j \in J_{i}} \sum_{l \in L} \frac{1}{t_{l}^{t u r n}} e_{l i j} x_{l i j} \\
\text { minimise } \sum_{(r, s) \in Q} \sum_{a \in A} t_{a} y_{a}^{r s}
\end{gathered}
$$

subject to

$$
\begin{aligned}
& \sum_{l \in L_{a}} \sum_{i \in I} \sum_{j \in J_{i}} \frac{1}{t_{l}^{t u r n}} x_{l i j} k_{i j} \geq \sum_{(r, s) \in Q} y_{a}^{r s} \text { for } a \in A \\
& \sum_{a \in A_{r}^{+}} y_{a}^{r s}=p^{r s} \quad \text { for }(r, s) \in Q \\
& \sum_{a \in A_{s}^{-}} y_{a}^{r s}=p^{r s} \quad \text { for }(r, s) \in Q \\
& \sum_{a \in A_{v}^{-}} y_{a}^{r s}=\sum_{a \in A_{v}^{+}} y_{a}^{r s} \quad \text { for }(r, s) \in Q, v \in N, v \neq r, v \neq s \\
& \sum_{i \in I} z_{l i} \leq 1 \quad \text { for } l \in L \\
& \sum_{j \in J_{i}} x_{l i j} \leq M z_{l i} \quad \text { for } l \in L, i \in I \\
& \sum_{l \in L} x_{l i j} \leq n_{i j} \quad \text { for } i \in I, j \in J_{i} \\
& y_{a}^{r s} \geq 0 \quad \text { for }(r, s) \in Q, a \in A \\
& x_{l i j} \in Z_{0}^{+} \\
& \text {for } l \in L, i \in I, j \in J_{i} \\
& z_{l i} \in\{0,1\} \\
& \text { for } l \in L, i \in I
\end{aligned}
$$

The optimisation criteria modelled by objective functions (3)-(6) were described in section 2. Objective functions (3)-(6) express investment costs, operating costs, emissions, and travelling times respectively. Constraints (7) ensure that the total number of passengers travelling through link $a$ does not exceed the capacity of vehicles operating on that link. Constraints (8) and (9) respectively ensure that the travel demand will be satisfied. Constraints (10) are flow conservation equations in nodes of the transportation network. Constraints (11) and (12) say that if line $l$ is chosen to operate then it will be served by exactly one vehicle type. Here constant $M$ is a big positive number, which does not restrict the number of vehicles of type $i$ assigned to line $l$. Constraint set (13) 
limits the number of vehicles assigned to line $l$ to the available amount. The remaining obligatory constraints (14)-(16) specify variable domains of definition.

\section{Solution process}

The solution methodology proposed in this research was inspired by approach published in [7]. It consists of two stages. In the first stage, the multiple objective program (MOP) described in the previous section is solved using a scalarization

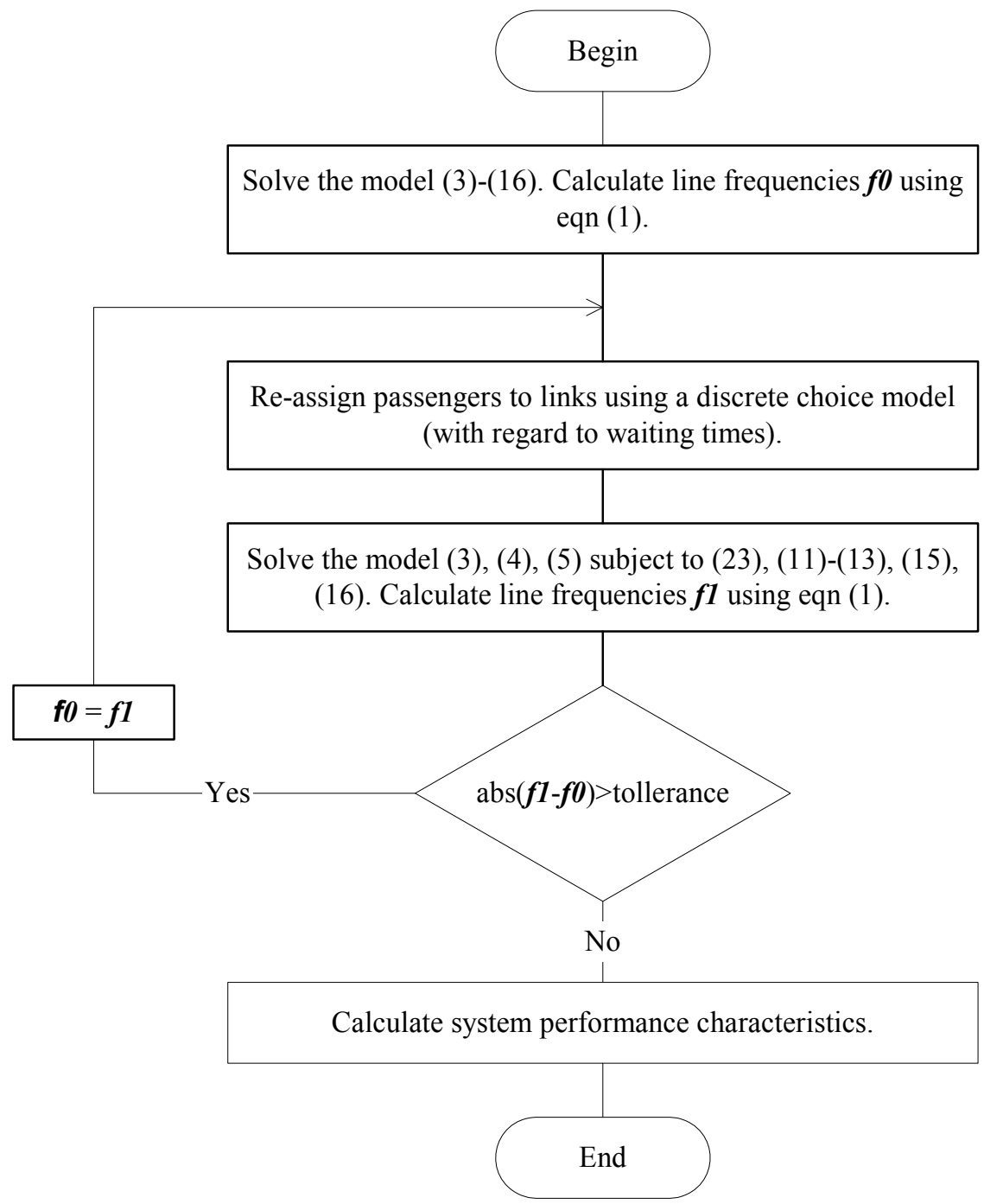

Figure 1: $\quad$ Flow chart of the solution process. 
method. The second stage consists of trip assignment and frequency setting. The process is outlined in Fig. 1 and detailed in the following text.

Assuming the initial set of candidate lines has been proposed, first the MOP (3)-(16) is solved.

Scalarization is a traditional approach to solving MOPs, which combines in general $m$ objective functions $f_{1}, \ldots, f_{m}$ to a single objective function by means of a scalarizing function. There are several scalarization techniques. One of them uses a reference point in the objective space [5]. A reference point is typically a vector of satisfactory or desirable criterion values. In case the analyst does not know desirable criterion values, the ideal point may be used as a reference point. The ideal point is such a point in the objective space, whose coordinates $f_{i}^{*}$ are the best values of particular objective functions:

$$
f_{i}^{*}=\min \left\{f_{i}(\boldsymbol{x}): \boldsymbol{x} \in X\right\} \text { for } i=1, \ldots, m
$$

where $X$ is a set of feasible solutions.

The single objective problem (SOP) corresponding to the given MOP minimises a weighted distance between the MOP solution and the ideal point. If $w_{i} \geq 0$ is the weight of the $i$-th objective function deviation, then the SOP is formulated as:

$$
\begin{aligned}
& \min \left(\sum_{i=1}^{m}\left(w_{i}\left(f_{i}(\boldsymbol{x})-f_{i}^{*}\right)\right)^{p}\right)^{1 / p} \\
& \text { subject to } \boldsymbol{x} \in X .
\end{aligned}
$$

For $p=\infty$ we get so-called Tchebycheff-norm scalarization, where the objective function (18) takes the form:

$$
\min \max _{i=1, \ldots, m}\left\{w_{i}\left(f_{i}(\boldsymbol{x})-f_{i}^{*}\right)\right\}
$$

If all the objective functions are of the same importance, weights $w_{1}, \ldots, w_{m}$ can normalize the deviations of the functions from the ideal point [9]. To derive weights this way, we need to know so-called Nadir point. Nadir point is such a point in the objective space, whose coordinates $f_{i}^{N}$ are the worst values of particular objective functions:

$$
f_{i}^{N}=\max \left\{f_{i}(\boldsymbol{x}): \boldsymbol{x} \in X_{E}\right\} \text { for } i=1, \ldots, m
$$

where $X_{E}$ is a set of effective solutions. If $c_{i j}$ is a coefficient multiplying variable $x_{j}, j=1, \ldots, \mathrm{n}$ in the $i$-th objective function, then a normalizing coefficient $a_{i}$ is defined by eqn (21):

$$
a_{i}=\frac{f_{i}^{N}-f_{i}^{*}}{f_{i}^{I}} \cdot \frac{1}{\sqrt{\sum_{j=1}^{n} c_{i j}^{2}}} \text { for } i=1, \ldots, m
$$


and used to derive weight $w_{i}$ :

$$
w_{i}=\frac{a_{i}}{\sum_{i=1}^{m} a_{i}}
$$

In our line-planning problem, we have four-dimensional objective space, where functions $f_{1}, \ldots, f_{4}$ correspond to objective functions (3), (4), (5), and (6) respectively. The solution of the problem (19) subject to (7)-(16) specifies how many vehicles of the given type and size are allocated to the lines. Lines, which are not assigned a vehicle, will not operate. The line frequencies are derived from the number of vehicles using eqn (1).

At the same time, the solution specifies optimal passengers' routes in the line network. Since the model minimizes system performance measures, some passengers may be forced to take very long routes possibly with a lot of transfers because it is inefficient to establish lines covering shortest paths for weak flows.

In the second stage of the solution process we try to improve the solution so that it can respect passengers' behaviour in case there are multiple alternative paths between a given origin-destination pair. In this stage the line frequencies are adjusted repeatedly until the internal consistency of frequencies is achieved.

The stage involves an iterative process consisting of trip assignment and solution of a simplified mathematical programming model.

First a new assignment of travel demand to the proposed set of lines is computed using a discrete choice model [10]. This process results in new passenger flows on the links.

Then a modified MOP based on re-computed flows is solved. Now the model does not solve the routes of passengers, it only computes the number of vehicles to be assigned to lines, i.e. line frequencies. It means that the modified model does not contain variables $\boldsymbol{y}$, objective (6), constraints (8)-(10) and (14), and constraints (7) obtain the following form:

$$
\sum_{l \in L_{a}} \sum_{i \in I} \sum_{j \in J_{i}} \frac{1}{t_{l}^{t u r n}} x_{l i j} k_{i j} \geq q_{a} \quad \text { for } a \in A
$$

where $q_{a}$ is the total flow on link $a$. By solving the model (3), (4), (5) subject to (23), (11)-(13), (15), (16) we get new frequencies. These new frequencies are compared with the previous ones and if they differ by more than a user-defined tolerance then the second stage repeats.

The trip assignment procedure should be described more precisely. It considers each origin-destination pair separately. For a given $(r, s)$ couple, all feasible paths in the line network from stop $r$ to $s$ are found. Feasibility of a path is determined by two conditions: first, the path is a direct path or it contains at most a user-defined number of transfers; second, the travel time along this path (including waiting times) does not exceed the minimum travel time by a specified threshold. In case the headways are less than 10 minutes, the average passenger waiting time can be estimated using half headway model $[4,7,8]$. 
Otherwise an analytical model derived in [8] can be used. The trip assignment model comes into the play if there are more feasible paths. In such a situation, a utility-based choice model can be used to predict the number of passengers who take a particular path. A traveller chooses a path with certain probability, which depends on the path utility. The utility has a deterministic and a random portion. The deterministic portion represents an observed measure of the path, while the random portion represents the randomness in the traveller's behaviour, i.e. that component of the utility which is unknown to the analyst. In the context of urban public transport we can suppose that all feasible paths with the same origin and destination have the same cost. So only the travel time represents the deterministic part of the path utility. From the utility-based choice model family, the multinomial logit model can be used in the context of path choice. Let $K(r, s)$ denote the set of all feasible paths from $r$ to $s$ and $t_{i}^{\text {path }}$ stand for the travel time on path $i$. Then the number of passengers who take path $k$ on their trip from $r$ to $s$ is defined by eqn (24). Negative travel time in eqn (24) indicates that increased travel time reduces the utility of a path.

$$
p_{k}^{r s}=\frac{\exp \left(-t_{k}^{\text {path }}\right)}{\sum_{i \in K(r, s)} \exp \left(-t_{i}^{\text {path }}\right)} p^{r s}
$$

Based on these path flows, link flow $q_{a}$ is computed as the sum of flows on all paths which go through link $a$.

After the iterative process has finished, system performance measures can be calculated. A decision maker is interested not only in criteria values, but also in other characteristics, such as the average in-vehicle time (per a travelling person), the total and average waiting time, the total and average number of transfers, the minimum, maximum and average line length expressed in number of links and number of kilometres, and so on.

\section{Conclusion}

In the paper we have described a multiple criteria mathematical programming model for the urban line network design and proposed a solution procedure. The solution procedure includes solving the model by a linear programming solver and adjustment of the solution using a discrete choice model to make the results more realistic. Verification of the model using real data for a middle-sized city in the Czech Republic will be presented at the conference.

\section{References}

[1] Blaton̆, M., Vícekriteriální optimalizace linek MHD. Proc. of the Seminar Discrete Optimisation Problems in Transportation. University of Pardubice: Pardubice, 2009. In Czech. 
[2] Borndörfer, R., Grötschel, M. \& Pfetsch, M.E., A column-generation approach to line planning in public transport. Transportation Science, 41(1), pp. 123-132, 2007.

[3] Ceder, A. \& Wilson, N.H., Bus network design. Transp. Research Part B, 20(4), pp. 331-344,1986.

[4] Černý, J. \& Kluvánek P., Základy matematickej teórie dopravy, VEDA: Bratislava, 1991. In Slovak.

[5] Ehrgott, M. \& Wiecek, M.M., Multiobjective programming (Chapter 17). Multiple Criteria Decision Analysis: State of the Art Surveys, eds. J. Figueira, S. Greco \& M. Ehrgott, Springer: New York, 2005.

[6] Erlander, S. \& Schéele, S., A mathematical programming model for bus traffic in a network. Proc. of the $6^{\text {th }}$ Int. Symposium On Transportation and Traffic Theory, ed. D.J. Buckley, Elsevier: New York, London, Amsterdam, pp. 581-605, 1974.

[7] Fan, W. \& Machemehl, R.B., Optimal Transit Route Network Design Problem: Algorithms, Implementations, and Numerical Results. Report No. SWUTC/04/167244-1. Center for Transportation Research, University of Texas at Austin, 2004.

[8] Fan, W. \& Machemehl, R.B., Characterizing bus transit passenger waiting times. $2^{\text {nd }}$ Material Specialty Conference of the Canadian Society for Civil Engineering. Canadian Society for Civil Engineering: Montreal, 2002.

[9] Fiala, P., Modely a metody rozhodování. Oeconomica: Praha, 2006. In Czech.

[10] Koppelman, F.S. \& Bhat, C., A Self Instructing Course in Mode Choice Modeling: Multinomial and Nested Logit Models, 2006, Online. http://www.civil.northwestern.edu/people/koppelman/PDFs/LM_Draft_060 131Final-060630.pdf

[11] Peško, Š., Podpora metód operačného výskumu pri navrhovaní systému liniek. Proc. of the $6^{\text {th }}$ Int. Conf. On Urban Transportation Infrastructure. University of Žilina: Žilina, 2008. In Slovak.

[12] Surovec, P., Provoz a ekonomika silnični dopravy I. Vysoká škola báňská: Ostrava, 2000. In Czech.

[13] Teichmann, D., O několika modifikacích matematického modelu přidělování vozidel linkám v městské hromadné dopravě. New Railway Technique, 17(1), pp. 20-23, 2009. In Czech. 